\title{
LADDER SHAFT SECONDARY DEVELOPMENT BASED ON SOLID WORKS
}

\author{
Jin Junsheng ${ }^{1}$, Yan Dong ${ }^{2}$ \\ ${ }^{1}$ College of Mechanical Engineering, Shanghai University of Engineering Science, China \\ ${ }^{2}$ College of Mechanical Engineering, Shanghai University of Engineering Science, China
}

\begin{abstract}
SolidWorks is a simple operation, easy to learn and easy to use software, in order to improve the work efficiency of mechanical design can use API function, it provides the secondary development of SolidWorks. With ladder shaft parameterized design as an example, this paper studies based on VB language of SolidWorks secondary development technique, the user can customize the parameters of the shaft, in the design of man-machine interface, the staff only need to input the specific related parameters, can be automatically generated ladder shaft.
\end{abstract}

Keywords: Ladder shaft; SolidWorks; Secondary development; VB; Parameterized - ***

\section{INTRODUCTION}

With the rapid advance of digital informatization process, now the three-dimensional CAD technology in more and more enterprises use. In order to reduce the cost in the design and production, shorten design cycle and enhance enterprise competitiveness, 3-d parametric technology should be born, with its closer to the modern concept of design and concurrent design thought has been more and more the favour of engineering design personnel, designers can in the early stages of development with 3 -d parametric technology rapid simulated 3-d sketch of the parts, then through numerical input constraints, the shape and size map precision parts, and parts to be included in the component library, convenient use and modification work. Thus greatly reduces the repetitive drawing time, improve the working efficiency. Based on 3-d CAD software SolidWorks as a platform for parameter secondary development methods and key technologies are discussed, through an example to illustrate in detail the second time the whole process of development.

Ladder shaft because of the characteristic of the easy parts and fixed installation, widely used in mechanical structure. Ladder shaft its structural parameters in the process of design and processing technology level and reliability of the size of the whole machine has a great influence. For the design of the stepped shaft is accomplished quickly, to design a software to replace the traditional manual drawing, it is necessary to improve design efficiency.

\section{THE BASIC PRINCIPLE OF SOLIDWORKS}

\section{SECONDARY DEVELOPMENT}

2.1 Two Forms of the Secondary Development of SolidWorks

Any support OLE (Object Linking and Embedding, objects connected with embedded) and COM (Component Object Model, and set up Object Model) interface programming languages can be used as a Solid Works development tools. SolidWorks secondary development is divided into two kinds: one kind is based on automation technology, can develop EXE application form.Another form of development is based on COM, this technique can be used most SolidWorks API (application programming interface).API can be controlled Solid Works session, generate *. DLL file format, is the Solid Works plug-in.

\subsection{The Object of the Secondary Development of}

\section{SolidWorks}

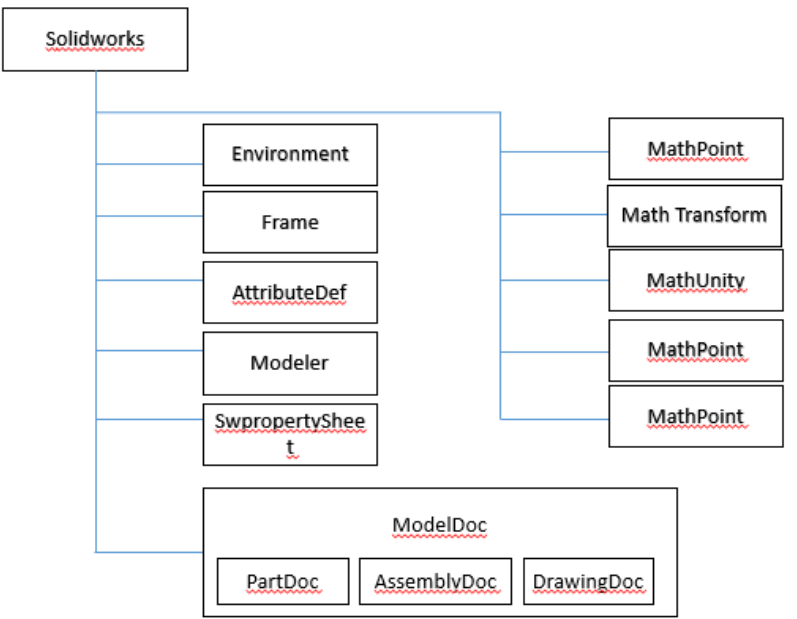

Fig -1: Object outline diagram of Solidworks 
Can see from figure 1 SolidWorks objects including Model Doc, the Environment, Frame, the Attribute Def, Modeler, SWProperty Sheet as well as some other object. SolidWorks is the highest object in SolidWorks API, it can directly or indirectly, access to all the other objects in the SolidWorks API. Using SolidWorks can realize the basic operation procedures, such as creating, opening, closing, end of the file, arrange ICONS and Windows, set the current activation file, generate attribute definition, can also set the system environment of Solid is Works.

SolidWorks contained in the object is divided into several layers, each layer and includes a number of objects, each object has its own properties, methods and events. Covers the entire SolidWorks OLE object data model, through to the SolidWorks OLE object attributes set and the OLE object method calls, you can in the user's own system to realize the same function as the SolidWorks.

\section{SECONDARY DEVELOPMENT TOOLS OF SOLIDWORKS}

Visual Basic is a kind of developed by Microsoft company, structured, modular, object-oriented, include to assist the development environment of event-driven mechanism for Visual programming language. Since VB introduced the concept of control, we can directly use many already write good VB program, greatly reducing the time from the programmers to develop, improve work efficiency.The feature of using $\mathrm{VB}$ to secondary development of SolidWorks, can quickly build your own application.

\section{SECONDARY DEVELOPMENT METHOD AND PROCESS OF SOLIDWORKS}

\subsection{Secondary Development Method of SolidWorks}

This paper mainly introduces SolidWorks secondary development based on VB language, on the secondary development with VB language and by two forms, one is a complete programming language development, the other is a combination of design variables and the programming language development. By API interface, the former need to design personnel to program written to describe all of the objects, so that we can realize $3 \mathrm{~d}$ graphics driver, and accurately size the three-dimensional modelling of complex structure. But this kind of development designers are required to be familiar with programming language, at the same time, because all objects need artificial expression definition, so there are design cycle is long, heavy workload, the problem of development efficiency is not high. The latter mainly provide macro recording in SolidWorks as a tool. Through macro operating on the program, the code used as engineering foundation, then carries on the appropriate edit, add to the application code. Then through the macro operation and interactive tasks, not only can improve the efficiency of programming, and more convenient for engineering designers. With that of the latter in the development process of obvious advantages, the development mode has become the main melody of the secondary development of SolidWorks, the following for the approach to elaborate the whole process of the secondary development of SolidWorks.

\subsection{Secondary Development Process of SolidWorks}

Application development process as shown in figure 2, which mainly includes the construction of three-dimensional model diagram and determine the relationship between size, Access database connections, user interface design, program code, write form event and debug program, etc.

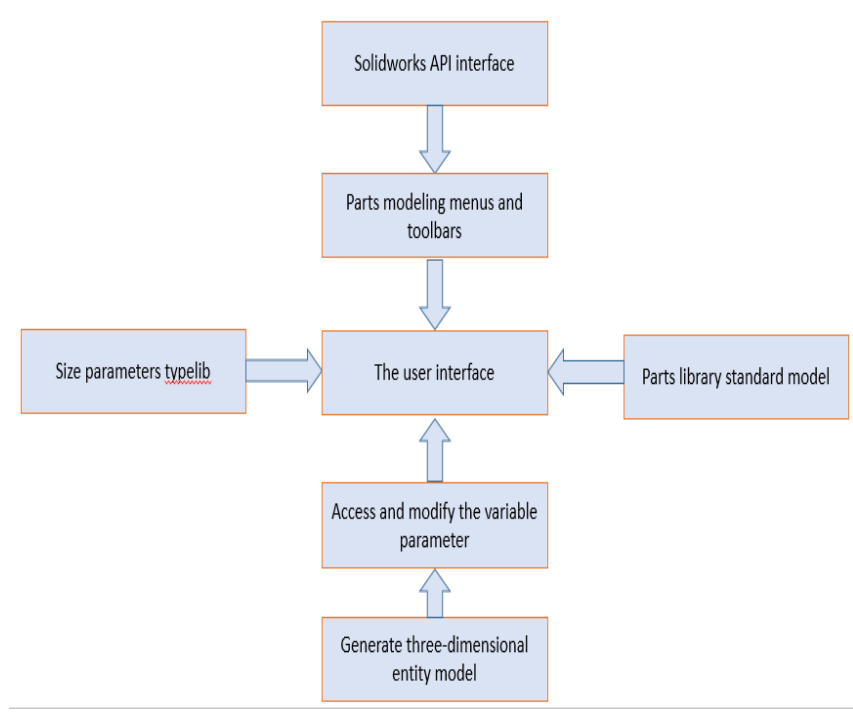

Fig -2: The flow chart of the SolidWorks secondary development

\section{LADDER SHAFT PARAMETRIC DESIGN}

\subsection{Use Macro to Record the Code}

By SolidWorks macros can record of SolidWorks users in the process of operating the click of a mouse, menu selection, and perform the operations such as keys, the record of operation process can also playback.

Generally there are two ways to draw the ladder shaft: one is to choose the generated by rotating convex platform ladder shaft, another it is to choose piecewise generate axial tensile entities.Here to record macro program is simple, choose generated by rotating convex platform ladder shaft, as shown in figure 3.

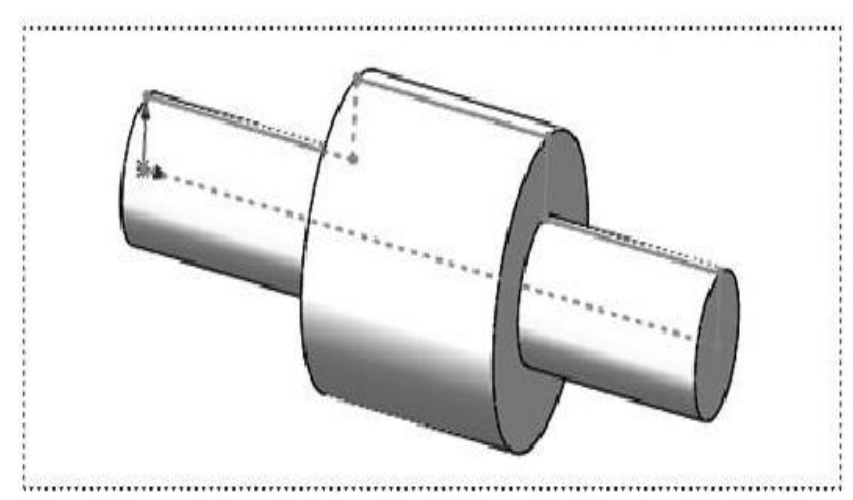

Fig -3: Figure of Stepped shaft parts 


\subsection{Add the Form to the Macro}

By the user is added in the macro forms, you can add a user interface for the application.Kit components on the board Label, the Command Button, Text Box control is added to the user form, and then in the properties window will control Command for the, the Command Button2 properties are set to "generate axial" cancel "As shown in figure 4.

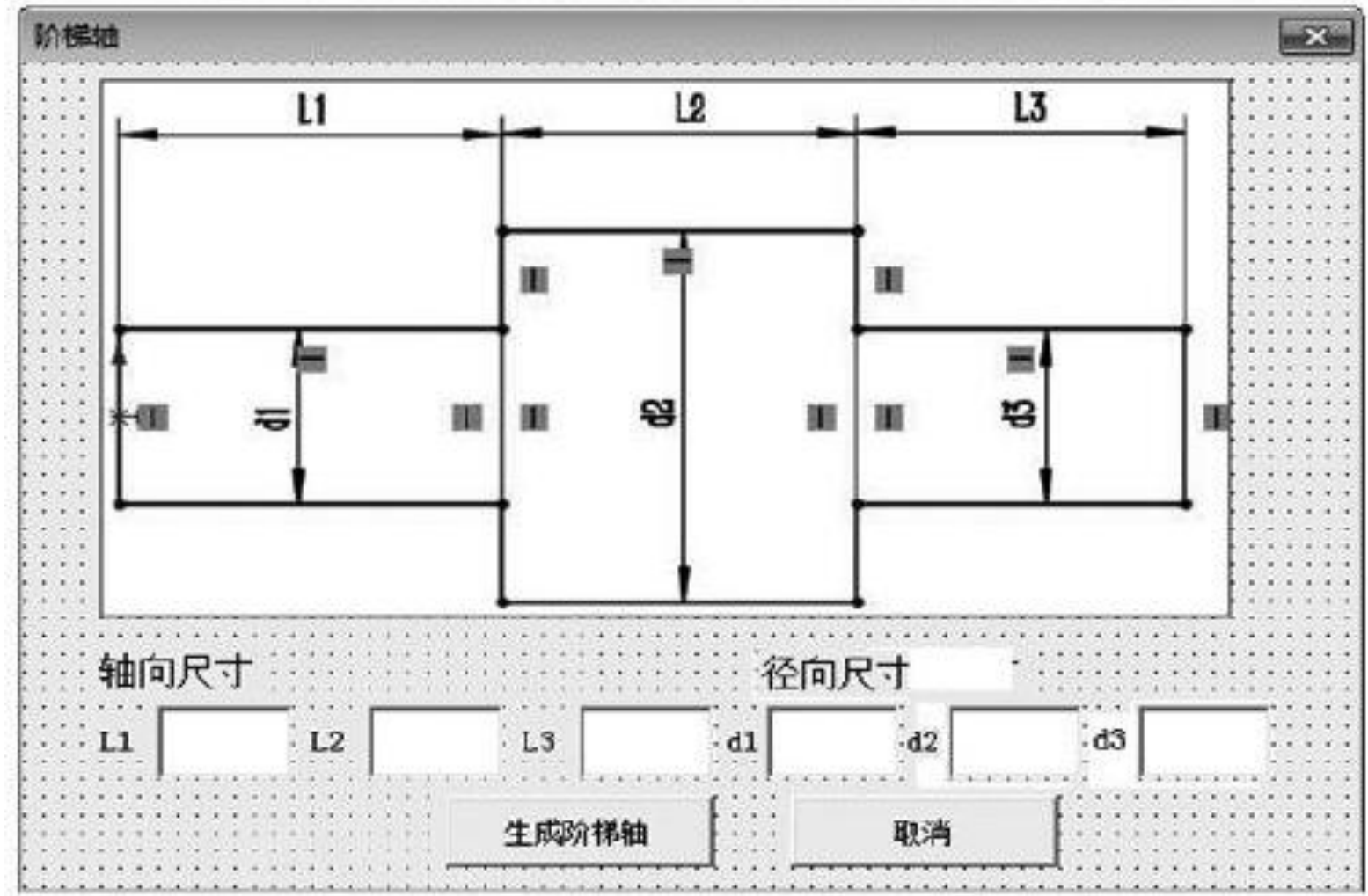

Fig -4: Figure of form

\subsection{Write the Program Code}

In SolidWorks, when after the completion of the macro recording, Solid Works themselves can generate macro code.Recorded in the macro code, you can find some to meet the requirements of our code, we can copy them to have to make up the program to use, again to write, modify, such as operation, finally set up their own programs, 3-d modeling of complete parts.

(1) Write a Solid Works associated with VB code

Dim swAppAs Object

Dim Part As Object

Set swApp= Create Object ("SldWorks.Application") swApp. Visible $=$ True

(2) Add code define a variable

Because when we create a new parts in SolidWorks, as the default units are in $\mathrm{mm}$ (millimeter), and we are through the human-computer interaction interface of the input value is indeed a m (meter), so pay attention to the unit in your application must be mutual transformation, all divided by 1000 .

Dim L1 As Double

Dim L2 As Double

Dim L3 As Double

Dim d1 As Double

Dim d2 As Double
Dim d3 As Double

$\mathrm{L} 1=\mathrm{TextBox} 1 / 1000$

$\mathrm{L} 1=\mathrm{TextBox} 2 / 1000$

$\mathrm{L} 1=\mathrm{TextBox} 3 / 1000$

$\mathrm{d} 1=$ TextBox $4 / 2000$

$\mathrm{d} 2=$ TextBox $5 / 2000$

$\mathrm{d} 3=$ TextBox $6 / 2000$

(3) Modify part of the main code

Sub main()

User Form1.Show

End Sub

(4) Edit "cancel" command code

Private Sub CommandButton2_Click()

End

End Sub

\section{CONCLUSION}

In this article, through the VB to secondary development of SolidWorks, designed the human-computer interaction interface, enter the ladder shaft design parameters, can be achieved in SolidWorks in the 3-d model automatically generate the ladder shaft, greatly reduce the design time, and can greatly improve the quality in the precision of parts in the process of traditional design, and the problem of low efficiency. 


\section{REFERENCES}

[1] HanSulan, Chen Ge. Based on VB Solid Works, the development of standard parts library research $[\mathrm{J}]$. Journal of north China institute of water resources and hydropower,2009,30(1):65-68.

[2] LiuLianying, Visual Basic programming tutorial [M]. Beijing: mechanical industry publishing house, 2011

[3] Ding Jie. Based on the Solid Works of machine parts parameterized design method [J]. Journal of gansu science and technology, 2009, 25 (3) : 21-24.

[4] SolidWorks company. Solid Works API secondary development [M]. Beijing: mechanical industry publishing house, 2010.

[5] Ye Xuezi, Chen Chaoxiang. SolidWorks advanced tutorial: secondary development and API [M]. Beijing: mechanical industry publishing house, 2009. 